AM BESTEN BEIDES SUBSTITUIEREN

\title{
Hartnäckige Eisenmangelanämie? Test auf subklinische Hypothyreose!
}

- Sprechen Patienten mit Eisenmangelanämie auf die Eisensubstitution nicht an, lohnt ein Schilddrüsencheck: Bei subklinischer Hypothyreose könnte es sinnvoll sein, Eisen mit Levothyroxin zu ergänzen.

Patienten mit einer Unterfunktion der Schilddrüse leiden relativ häufig an einer normochromen normozytären Anämie; sie kann durch die Einnahme von Levothyroxin behoben werden. Darüber hinaus scheint aber auch ein Zusammenhang zwischen einer (subklinischen) Hypothyreose und einer Eisenmangelanämie zu bestehen. Wie sich Hämoglobin und Ferritin unter diesen Umständen am besten normalisieren lassen, haben Ärzte der Universität Bushehr im Iran in einer randomisierten Doppelblindstudie untersucht.

60 Patienten mit Eisenmangelanämie (definiert als $\mathrm{Hb}<12 \mathrm{~g} / \mathrm{dl}$ und Ferritin $<25 \mu \mathrm{g} / \mathrm{l}$ für Frauen und als $\mathrm{Hb}<13,5 \mathrm{~g} / \mathrm{dl}$ und Ferritin $<30 \mu \mathrm{g} / \mathrm{l}$ für Männer) und subklinischer Hypothyreose (TSH 4,5-10 mU/l bei normalen fT3- und fT4-Werten) wurden dazu entweder mit Eisensalz plus Placebo $(n=20)$, mit Levothyroxin plus Placebo $(n=20)$ oder mit Eisensalz plus Levothyroxin behandelt. Nach dreimonatiger Therapie war nur bei den doppelt substitutierten Patienten ein signifikanter Anstieg von $\mathrm{Hb}$ und Ferritin messbar, und zwar um 1,17 g/dl bzw. um $10,78 \mu \mathrm{g} / \mathrm{l}$. Dadurch wurde in den meisten Fällen eine Normalisierung der Werte erreicht. Die Behandlung mit Eisen plus Schilddrüsenhormon war damit den Einzeltherapien hoch signifikant überlegen. Für den TSH-Spiegel spielte es dagegen keine Rolle, ob Levothyroxin allein oder in Kombination mit Eisen gegeben wurde, der Anstieg war in beiden Gruppen gleich groß.

Die Studienautoren um Dr. M. Ravanbod vermuten, dass eine Hypothyreose die Eisenresorption beeinträchtigt, weil weniger Magensäure gebildet wird, die zum Aufschluss des Eisenkomplexes aus pflanzlichen Quellen benötigt wird. „Levothyroxin kann diesen ungünstigen Effekt auf die Eisenresorption rückgängig machen."

Die Empfehlung der Autoren lautet daher: „Wenn die Eisensubstitution bei einer Ei-

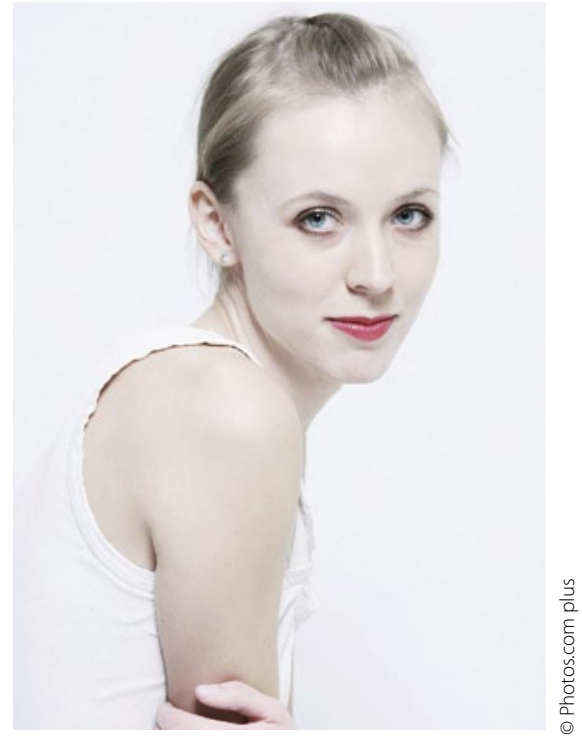

Warum ist sie so blass?

senmangelanämie nicht wirksam ist, ist es - besonders in Jodmangelgebieten - wichtig, eine subklinische Hypothyreose in Betracht zu ziehen."

Besteht außer der Eisenmangelanämie auch eine subklinische Hypothyreose, raten die iranischen Ärzte, beide Mangelzustände zu substituieren.

BS

- Ravanbod M et al. The American Journal of Medicine 2013; 126: 420-424

\section{ERHÖHTES STERBERISIKO}

\section{Eisenmangel: Gefahr für betagte Herzpatienten}

— Eine Anämie im Alter ist ein Symptom mit hohem Risikopotenzial. Bei Menschen jenseits der 65 verschlechtern zu niedrige Hämoglobinwerte nicht nur Krankheitsverläufe, sie sind auch mit häufigeren Klinikeinweisungen assoziiert und erhöhen vor allem das Sterberisiko.

Bei Herzinsuffizienzpatienten ist der Hämoglobinwert ein unabhängiger Risikofaktor für schwere Krankheitsverläufe, Klinikeinweisungen und Mortalität. Wie Dr. Gabriele Röhrig von der Klinik für Geriatrie im St.Marien-Hospital Köln betont, ist die Sterblichkeit am geringsten bei Hb-Werten zwischen 14,5 und 15,4 g/dl; bei Werten darunter und darüber steigt sie im Sinne einer Uförmigen Kurve. Die Überlebenschancen innerhalb eines Jahres sind für herzinsuffi- ziente Patienten mit Anämie deutlich geringer als für nicht anämische Herzkranke. Die häufigste Ursache für eine Anämie bei Herzpatienten ist der Eisenmangel. Dabei liegt bei der Mehrzahl der Patienten ein funktioneller Eisenmangel vor, d. h. eine gestörte Mobilisation von Eisen aus den körpereigenen Depots. Zugrunde liegt ein chronischer Entzündungsprozess, der oft mit der Herzinsuffizienz assoziiert ist. Der Ferritinwert ist dabei typischerweise normal oder sogar erhöht, während die Transferrinsättigung auf $<20 \%$ abfällt.

Eine Anhebung des Eisenspiegels kann die Leistungsfähigkeit von CHI-Patienten steigern, und zwar nicht nur bei anämischen Patienten. Röhrig zitiert hierzu die FAIR-HFStudie, eine prospektive Multicenterstudie mit über 400 Patienten in den NYHA-Stadien II oder III: Die Teilnehmer schnitten nach i. v. Eisensubstitution über 24 Wochen nicht nur im 6-Minuten-Gehtest deutlich besser $a b$, sondern profitierten auch mit einer signifikant höheren Lebensqualität.

Auch beim funktionellen Eisenmangel ist eine Eisensubstitution sinnvoll. Um einen $\mathrm{Hb}$-Anstieg um $1 \mathrm{~g} / \mathrm{dl}$ zu erreichen, sind etwa 150-200 mg Eisen erforderlich, so Röhrig. Primär wähle man die orale Substitution. Wenn sich damit der Eisenstatus nicht verbessern lässt oder die Präparate nicht vertragen werden, besteht die Indikation für eine parenterale Applikation.

$\mathrm{EO}$ -

- Röhrig G et al. Z Gerontol Geriat 2012; 45: 191-196; doi:10.1007/s00391-012-0320-8 